\title{
Predictores de mala salud autopercibida en una población de personas mayores
}

\author{
Trinidad Lorenzo, José Carlos Millán-Calenti, Laura Lorenzo-López, Alba Sánchez y \\ Ana Maseda
}

Grupo de Investigación en Gerontología, Departamento de Medicina, Facultad de Ciencias de la Salud, Universidad de A Coruña, A Coruña, España

\begin{abstract}
Resumen
Introducción: La salud autopercibida forma parte del concepto global de «calidad de vida», además de ser una medida válida para conocer el estado de salud. El objetivo principal de este trabajo es evaluar el efecto predictor de ciertas variables sobre la mala salud autopercibida en una población de personas mayores.

Material y métodos: Se lleva a cabo un estudio transversal con una muestra de 140 personas mayores. Mediciones: edad, género, estudios, hábitat, estado cognitivo, discapacidad física, enfermedades, percepción de la salud y apoyo social. Para analizar el efecto de las diferentes variables sobre la mala salud autopercibida se aplicó un modelo de regresión logística y una curva ROC para establecer los valores de corte de dichas variables, con la mejor sensibilidad y especificidad para la predicción de mala salud autopercibida.

Resultados: Se observa una asociación significativa entre una mala salud autopercibida con la edad, la comorbilidad, y con la percepción de apoyo social funcional débil, mientras que no existía asociación con el género, el hábitat o el nivel educativo.

Conclusiones: La edad elevada, el número de enfermedades diagnosticadas, y el apoyo social funcional son los factores de riesgo de la salud autopercibida, mientras que las características y repercusiones delas enfermedades no deben ser consideradas.
\end{abstract}

Palabras clave: Salud autopercibida, Comorbilidad, Apoyo social, Envejecimiento

\section{Predictors of poor Self-Rated Health in an elderly population}

\begin{abstract}
Introduction: Self-Rated Health is part of the comprehensive concept of Quality of Life and is a valid measurement of health status. The main objective of this study was to test the predictive value of some different variables on the poor Self-Rated Health among elders.

Material and methods: We performed a cross-sectional study on a sample consisting of 140 participants. Measurements: age, gender, level of education, environment, cognitive status, physical impairment, diseases, health perception and social support. The influence of the studied variables on the poor Self-Rated Health was performed with a logistic regression analysis and a ROC curve to establish the cut-off values for these variables with the best sensitivity and specificity to predict the poor Self-Rated Health.

Results: A poor Self-Rated Health was significantly associated with age, comorbidity, and the perception of poor functional social support, whereas no association was found with gender, environment and educational level.

Conclusions: Old age, the number of diagnosed diseases, and functional social support are Self-Rated Health risk factors, while the characteristics and repercussions of the diseases should not be considered.
\end{abstract}

Keywords: Self-Rated Health, Comorbidity, Social support, Aging 


\section{Introducción}

La salud autopercibida (SAP) mediante la pregunta «En general, ¿cómo calificaría usted su salud en los últimos 12 meses?» establece una forma sencilla, directa y global de valorar el estado de salud $^{1}$.Utilizada mayoritariamente en las encuestas de salud ${ }^{1}$, incluso en las personas mayores de 65 años, recoge una amplia información sobre los aspectos de la salud más allá de la ausencia o presencia de enfermedad, o mediante el historial clínico².

La SAP se asocia con factores sociodemográficos como la edad y el género, si bien la naturaleza y el carácter de esta asociación no está definida claramente ${ }^{3-5}$. También se ha analizado la relación entre la SAP y las enfermedades crónicas ${ }^{5}$, y su valor predictivo ante la morbimortalidad ${ }^{2}$. Además, con relación a las características bio-psico-sociales ${ }^{6}$ del ser humano es importante analizar las complejas relaciones entre el apoyo social y la SAP en las personas mayores. El apoyo social puede influir en comportamientos negativos sobre la salud, peor bienestar mental y físico; y, además, el apoyo social pobre se ha relacionado con mayores tasas de mortalidad ${ }^{7,8}$.

Partiendo de la hipótesis de que la SAP es el resultado de una serie de factores y, que conociéndolo podríamos llegar a predecirla y, por tanto, prevenirla, los objetivos del estudio se dirigen a establecer la relación de la SAP con las características sociodemo-gráficas (edad, género, nivel educativo y hábitat), comorbilidad y apoyo social.

\section{Material y métodos}

Se realizó un estudio transversal con 140 personas seleccionadas mediante muestreo no probabilístico por casos consecutivos, que debían cumplir como criterios de inclusión ser mayores de 65 años, presentar deterioro psíquico, funcional y/o sensorial y haber vivido al menos 12 años en un municipio rural gallego (Mondoñedo, Lugo) o urbano (A Coruña). Se contactó con los participantes mediante las Áreas de Servicios Sociales de sus ayuntamientos.

La presencia de deterioro, para las 3 variables de salud establecidas, fue evaluada por terapeutas debidamente entrenados. El estado cognitivo se evaluó mediante el Mini-Examen Cognoscitivo (MEC), adaptado y validado a la población española. Se excluyeron aquellos sujetos con puntuación menor de 24, ya que su nivel de deterioro podría interferir en la capacidad para responder a los cuestionarios. La dependencia para realizar las actividades básicas de la vida diaria se evaluó mediante el índice de Barthel ${ }^{10}$. Se incluyeron los participantes con una puntuación menor de 100 (menos de 90 si utilizaban silla de ruedas). Por último, los participantes informaban sobre los posibles trastornos auditivos o visuales. Los sujetos seleccionados fue-ron informados acerca del proyecto, otorgando su consentimiento informado.

Se creó un cuestionario estructurado mediante información proveniente de instrumentos consolidados $^{11,12}$ :

a) Datos sociodemográficos: género, hábitat, edad, nivel educativo y estado civil.

b) Autopercepción de la salud: «En general, ¿cómo calificaría usted su salud en los últimos 12 meses?» (muy buena, buena, regular, mala y muy mala). Para el análisis de respuestas, se dicotomizó la SAP en 2 categorías: «buena» (muy buena y buena), y «mala»(regular, mala y muy mala).

c) Existencia/ausencia de enfermedades crónicas diagnosticadas en los últimos 12 meses: por el médico de cabecera, solicitándose al posible participante un informe médico, historial clínico o similar. Enfermedades crónicas incluidas: artritis y/o reumatismo, trastornos visuales, auditivos, respiratorios, digestivos, mentales o genitourinarios, enfermedades cardíacas, diabetes, problemas de memoria, hipertensión, depresión y/o tristeza, enfermedad de Alzheimer o Parkinson, insomnio y cáncer. En función de los datos se creó la variable comorbilidad, recodificada en 2 niveles (presencia o no) a partir de la mediana, $\leq 4$ o más de 4 enfermedades.

d) Apoyo social: tanto funcional (percepción de apoyo), como estructural (tamaño de la red de apoyo). 


\section{Análisis estadístico}

Para establecer la asociación entre las variables estudiadas y la SAP dicotomizada se realizó un análisis de contingencias. Solo aquellas variables con relación estadísticamente significativa con la SAP se incluyeron en el análisis de regresión logística múltiple, identificándose qué variables independientes modificaban la SAP: edad $(p=0,000)$, comorbilidad $(p=0,003)$, apoyo social estructural $(p=0,021)$ y apoyo social funcional $(\mathrm{p}=0,000)$. Se estableció un nivel de significación de $\mathrm{p}<0,05$. Por último, se desarrolló una curva Receiver Operating Characteristic (ROC) para evaluar la exactitud dela prueba diagnóstica, es decir, su capacidad para clasificar con la mejor sensibilidad y especificidad a los participantes en categorías (buena/mala SAP), en relación con los valores de corte de variables demográficas, comorbilidad y apoyo social.

\section{Resultados}

La media de edad de los participantes fue de 79,5 años $( \pm 7,2)$.El $57,1 \%$ fueron mujeres y el $71,4 \%$ residían en entorno urbano. Con respecto al nivel educativo, el 10,6\% eran iletrados; el 28,6\% no tenían estudios, pero sabían leer y escribir; el 22,9\% tenían estudios primarios; el 25\% secundarios, y el 12,9\% habían recibido educación superior. Por estado civil, el 45\% eran viudos; el 36,4\%, casados; el 14,3\% solteros, y el 4,3\%, separados o divorciados.

La distribución de la SAP muestra que los varones (85\%) calificaron su salud peor que las mujeres (78,8\%), y los que vivían en entornos rurales (90\%) peor que los participantes urbanos (78\%). En relación con el nivel educativo, los 3 grupos indicaron mala SAP: iletrados (80\%), estudios primarios y secundarios $(82,1 \%)$ y educación superior $(83,3 \%)$.

Las enfermedades asociadas estadísticamente con la SAP fueron los problemas respiratorios, el insomnio y los trastornos genitourinarios. Se observó la relación existente entre el número total de enfermedades crónicas y la SAP ( $\mathrm{p}<0,05$ ). Por último, respecto a las variables de apoyo social, tanto el apoyo social funcional como el estructural tenían una relación estadísticamente significativa con la SAP.

La tabla 1 presenta resultados del análisis de regresión logística para predecir la SAP muy mala, mala o regular, después del ajuste por las variables sociodemográficas, la comorbilidad y el apoyo social funcional y estructural. La comorbilidad, asociada con la SAP ( $p<0,0001)$ en el análisis univariante, tenía el mismo efecto que la mediana del número de enfermedades crónicas $(\mathrm{p}=0,003)$ en el análisis multivariado. El predictor más fuerte para mala SAP fue lacomorbilidad.

El riesgo de tener mala percepción de la salud se incrementaba significativamente en 5,9 veces (IC 95\%: 1,972-17,375) si los individuos tenían una o más enfermedades significativas en el análisis univariante; el riesgo de mala SAP aumentó 1,1 veces (IC 95\%: 1,028-1,213) por cada año de incremento de la edad y, por último, el apoyo social funcional fue también buen predictor de pobre SAP (1,2 veces; IC 95\%: 1,048-1,399), no observándose relación significativa con el apoyo estructural.

La figura 1 muestra la curva ROC, que establece la probabilidad de tener mala SAP en función de los 3 predictores más fuertes. El área muestra que si escogemos un individuo al azar con mala SAP, la probabilidad de que tenga, por ejemplo, más edad que cualquier sujeto elegido al azar con buena salud es del 70,7\%. La sensibilidad y la especificidad indicaron que estas 3 variables $(p<0,05)$ eran capaces de predecir mala SAP. 
Tabla 1

Análisis de regresión logística para predecir una mala autopercepción de la salud, después del ajuste con aquellas variables (edad, comorbilidad y apoyo social funcional y estructural) relacionadas significativamente en un análisis de contingencia previo

\begin{tabular}{|c|c|c|c|c|c|}
\hline Variable & Coeficiente (B) & Valor de $\mathrm{p}$ & Odds ratio & Inferior & Superior \\
\hline Género (femenino vs. masculino) & $-0,211$ & 0,715 & 0,810 & 0,260 & 2,518 \\
\hline Hábitat (urbano o rural) & $-0,426$ & 0,563 & 0,653 & 0,154 & 2,770 \\
\hline Nivel educativo (iletrados vs. educación superior) & $-0,249$ & 0,771 & 0,779 & 0,146 & 4,170 \\
\hline Comorbilidad & 1,767 & 0,001 & 5,854 & 1,972 & 17,375 \\
\hline Apoyo social estructural & $-0,006$ & 0,896 & 0,994 & 0,908 & 1,089 \\
\hline Apoyo social funcional & 0,192 & 0,009 & 1,211 & 1,048 & 1,399 \\
\hline Edad & 0,110 & 0,009 & 1,117 & 1,028 & 1,213 \\
\hline
\end{tabular}

B: coeficiente de regresión.

\section{Discusión}

Nuestros resultados constatan que las personas etiquetan deforma subjetiva la/s enfermedad/es padecidas desde el punto de vista de la comorbilidad, con una base más cuantitativa(número de enfermedades), que cualitativa (características y repercusiones de las mismas). La SAP no empeora debido al diagnóstico por sí mismo, sino que podría verse influenciada por manifestaciones somáticas ${ }^{13}$, o procesos psicológicos en curso al suponer un empeoramiento en el bienestar «diario» del individuo. Nuestro hallazgo de que la comorbilidad predice mala SAP es consistente con otros estudios que, o utilizan mediciones válidas de comorbilidad (índice de comorbilidad de Charlson) o encuentran correlaciones significativas entre la SAP y la comorbilidad, creada a partir de enfermedades diagnosticadas ${ }^{14}$.

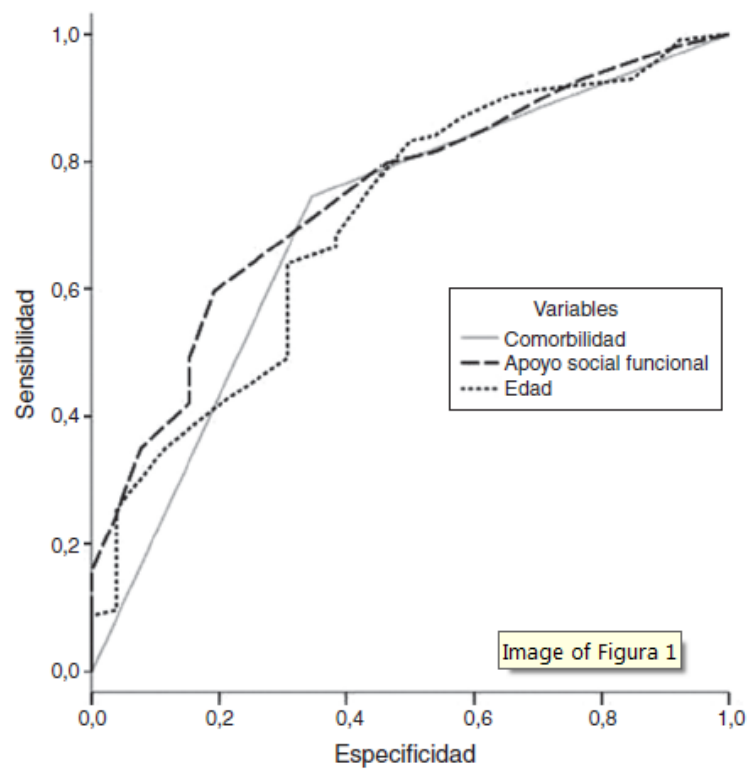

Figura 1. Curva ROC para predecir la probabilidad de tener muy mala, mala o regularsalud autopercibida, según la comorbilidad, el apoyo social funcional y la edad.Área bajo la curva: Edad: 0,707; Apoyo social funcional: 0,744; Comorbilidad: 0,700. 
Si bien existen resultados similares sobre la relación entre la SAP y la edad, este estudio ayuda a describir la naturaleza de dicha relación en la población mayor española. Al incrementarse la edad, los individuos no solo se adaptan con mayor facilidad a sus enfermedades preexistentes, sino que también valoran su salud dentro de las expectativas asociadas a su edad ${ }^{14}$, es decir, es de esperar el tener varias enfermedades. Existe pues, una concepción de la salud particular, vinculada a múltiples trastornos, y diferente a la poseída antes de envejecer.

Además de la relación con la comorbilidad, existiría una clara influencia del apoyo social funcional sobre la SAP en las personas de edad avanzada con algún tipo de deterioro. El apoyo social funcional también fue encontrado como variable predictora de mala SAP. Si bien diversos estudios ${ }^{1,2}$ muestran que los sujetos que sobrestimaban su salud empleaban criterios emocionales en lugar de médicos, la particularidad del estudio radica en el tipo de muestra, personas de 65 y más años con deterioro físico, cognoscitivo y/o social.

Señalar como limitaciones a esta investigación, la necesidad de realizar estudios longitudinales para conocer el grado de afectación de dichas variables con el paso del tiempo; y de reproducir el estudio en una muestra representativa estratificada por nivel educativo, para poder confirmar la relación significativa o no con la SAP.

Edad, comorbilidad y bajo apoyo social son 3 variables a tener en cuenta en la evaluación que de su mala salud hacen las personas mayores. Si bien sobre la primera de las variables no se puede inter-venir, la promoción de la salud e instauración de redes de apoyo social podrían contribuir a mejorar la calidad de vida de este colectivo y, por tanto, aliviar la sobrecarga de los sistemas públicos de salud.

\section{Conflicto de intereses}

Los autores declaran no tener ningún conflicto de intereses.

\section{Bibliografía}

1. Idler EL, Benyamini Y. Self-rated health and mortality: A review of twenty-seven community studies. J Health Soc Behav. 1997;38:21-37.

2. Idler EL, Hudson SV, Leventhal H. The meanings of self-ratings of health: A qualitative and quantitative approach. Res Aging. 1999;21:458-76.

3. Gallicchio L, Hoffman SC, Helzlsouer KJ. The relationship between gender, social support, and health-related quality of life in a community-based study in Washington County, Maryland. Qual Life Res. 2007;16:777-86.

4. Alves LS, Rodrigues RN. Determinantes da autopercepcão de saúde entre idosos do Município de São Paulo. Brasil Rev Panam Salud Pub. 2005;17:333-41.

5. Bjorner JB, Kristensen TS. Multi-item scales for measuring global self-rated health. Investigation of construct validity using structural equations models. Res Aging. 1999;21:417-39.

6. Millán-Calenti JC. Gerontología y Geriatría. En: Millán-Calenti JC, editor. Principios de Geriatría y Gerontología. Madrid: McGraw-Hill Interamericana; 2006.p. 3-20.

7. Kawachi I, Berkman LF. Social ties and mental health. J Urban Health.2001;78:458-67.

8. Eng PM, Rimm EB, Fitzmaurice G, Kawachi I. Social ties and change in social ties in relation to subsequent total and causespecific mortality and coronary heart disease incidence in men. Am J Epidemiol. 2002;155:700-9.

9. Lobo A, Saz P, Marcos G, Día JL, de la Cámara C, Ventura T, et al. Revalidacióny normalización del Mini-Examen Cognoscitivo (primera versión en castellano del Mini-Mental Status Examination) en población general geriátrica. Med Clin(Barc). 1999;112:767-74.

10. Mahoney FI, Barthel D. Functional evaluation: The Barthel Index. Md Med J.1965;14:56-61

11. Instituto de Mayores y Servicios Sociales. Encuesta de condiciones de vida de los mayores. [Internet] 2010 [consultado 3 Dic 2011]. Disponible en: http://www.imsersomayores.csic.es/estadisticas/encuestas/index.html

12. Ministerio de Salud y Política Social. Encuesta Nacional de Salud. [Internet] 2006 [consultado 13 Dic 2007]. Disponible en: http://www.msps.es/estadEstudios/estadisticas/encuestaNacional/encuesta2006.htm

13. Benyamini Y, Idler EL, Leventhal H, Leventhal EA. Positive affect and function as influences on self-assessments of health: Expanding our view beyond illness and disability. J Gerontol B Psychol Sci Soc Sci. 2000;55:107-16.

14. Heller DA, Ahern FM, Pringle KE, Brown TV. Among older adults, the responsiveness of self-rated health to changes in Charlson comorbidity was moderated by age and baseline comorbidity. J Clin Epidemiol. 2009;62:177-87. 